\title{
Essais
}

Revue interdisciplinaire d'Humanités

Politicité de la littérature et des arts contemporains

\section{La poésie documentaire, fabrique subversive du regard politique : Ce qui nous regarde, de Myriam Marzouki}

\section{Chloé Dubost}

\section{(2) OpenEdition Journals \\ Édition électronique \\ URL : http://journals.openedition.org/essais/1128 \\ DOI : 10.4000/essais. 1128 \\ ISSN : 2276-0970 \\ Éditeur \\ École doctorale Montaigne Humanités}

\section{Édition imprimée}

Date de publication : 15 septembre 2020

Pagination : 83-92

ISBN : 979-10-97024-08-6

ISSN : 2417-4211

Référence électronique

Chloé Dubost, « La poésie documentaire, fabrique subversive du regard politique : Ce qui nous regarde de Myriam Marzouki », Essais [En ligne], 16 | 2020, mis en ligne le 28 septembre 2020, consulté le 23 avril 2021. URL : http://journals.openedition.org/essais/1128; DOI : https://doi.org/10.4000/essais. 1128 


\section{La poésie documentaire, fabrique subversive du regard politique : Ce qui nous regarde, de Myriam Marzouki}

\section{Chloé Dubost}

La Compagnie du dernier soir, fondée par Myriam Marzouki ${ }^{1}$ en 2002, privilégie des textes contemporains dont le matériau linguistique, narratif et textuel est noué à des préoccupations politiques : United Problems of Cồt de la Main d'ouvre de Jean-Charles Massera, Europeana. Une brève histoire du $X X^{e}$ siècle de Patrick Ourednik, et Laissez-nous juste le temps de vous détruire d'Emmanuelle Pireyre maintiennent ainsi une hybridité certaine entre la forme de l'essai politique et celle de l'essai poétique. Depuis 2013, et après l'adaptation du roman Le Début de quelques choses d'Hugues Jallon², un cycle semble néanmoins s'être clos : l'écriture, plus personnelle, s'appuie dorénavant sur le montage de différentes matières documentaires, pour éclairer un fait social contemporain.

C'est dans cette nouvelle perspective que s'inscrit Ce qui nous regarde: créé en 2016, le spectacle répond aux vives polémiques suscitées par divers événements politiques qui ont rendu problématique l'interprétation et la réception, en France, de l'objet qu'est le voile. Nous les résumons ici : en 1989, lors de "l'affaire du voile », deux élèves du collège Gabriel-Havez (Creil) sont exclues parce qu'elles refusent d'ôter leur voile en classe ; en 2004, le gouvernement de Jacques Chirac interdit la monstration de tout signe religieux au sein de l'espace public; en 2010, le voile intégral est interdit ; l'été 2016, l'affaire du " burkini » a révélé à quel point le voile est systématiquement perçu comme le symbole d'une oppression patriarcale et comme le signe revendiqué d'un islam politique radicalisé et anti-occidental - qu'il s'agit, pour l'État, de combattre.

1 Myriam Marzouki, franco-tunisienne, se présente au début du spectacle comme " athée et féministe ". Ancienne élève de l'ENS Ulm et agrégée de philosophie, formée au théâtre au Conservatoire de Chaillot et au sein de divers théâtres universitaires, elle mène de front sa carrière d'enseignante et ses activités artistiques.

2 Hugues Jallon, Le Début de quelque chose, Paris, Gallimard, 2011. 
L'enjeu du spectacle de Myriam Marzouki est précisément de mettre en perspective cet imaginaire, et de révéler la manière dont notre regard sur le voile est fabriqué culturellement et socialement par l'intériorisation de différentes "narrations " médiatiques et politiques. Le spectacle est ainsi fondé sur la rencontre surprenante entre des documents hétérogènes : différentes représentations du voile sont proposées au spectateur, et leurs processus narratifs sont analysés puis déconstruits, pour ouvrir à des interprétations et perceptions inédites. La forme esthétique qu'est le montage documentaire apparaît alors comme une formalisation politique alternative : en confrontant différentes textures documentaires, la poésie rendrait hybrides et inattendus les signifiés conventionnels déployés par les représentations ; en déplaçant les pratiques discursives et sensorielles (discours, percepts, affects) construits par les documents, la poésie documentaire re-symboliserait l'imaginaire qui ratifie l'existence politique du voile. Le spectacle proposerait donc des contre-narrations, qui ne définissent pas l'objet, mais maintiennent un écart avec les imaginaires intériorisés et transfigurent la chronique conventionnelle - pour élargir le regard.

Le théâtre documentaire permet alors à la poésie de se défaire de l'éternelle opposition entre souci de la forme et souci du monde, qui lui confisque traditionnellement son pouvoir politiquement subversif : éloigné des notions de "théâtre poétique » ou de "poésie dramatique » dont la versification et le lyrisme seraient l'apanage, le "sentiment " poétique est ici issu de la forme documentaire et dicté par des " raisons politiques ${ }^{3}$ subversives, qui traversent et altèrent la $d o x a^{4}$, et élaborent ainsi un nouveau rapport symbolique et affectif avec l'objet-voile.

Or, l'imaginaire qui détermine le port du voile est symptomatique de la manière dont l'Occident légitime et inscrit le principe d'exclusion de certaines catégories de la population dans les cadres objectifs de la normalité : en subvertissant cet imaginaire, le spectacle contribue donc, par extension, à l'avènement d'une nouvelle rationalité politique et à une reconfiguration de la société postcoloniale, où les principes de normalisation et d'altérisation de la population sont repensés.

3 C'est avec ces mêmes expressions que Pier Paolo Pasolini justifie la réalisation du film $L a$ Rabbia (La Rage) en 1963. Cet artiste a nourri la dramaturgie du spectacle, comme nous aurons l'occasion de le constater plus avant.

4 Michel Foucault, Les Mots et les choses. Une archéologie du savoir, Paris, Gallimard, 2008, p. 13. 


\section{Formalisation poético-politique du monde}

L’un des premiers documents déployés par le spectacle est la Première Épître aux Corinthiens de Saint-Paul', qui est récitée par le comédien : pour les connaisseurs, ce texte permet de se souvenir que le port du voile n'est pas une exclusivité musulmane, mais aussi un dogme chrétien inscrit dans les textes fondateurs de la civilisation occidentale ; pour les autres, un temps certain est laissé au spectateur avant la révélation de la source du texte récité : le spectacle joue donc avec ce qui se produit lorsque la doxa occidentale, qui associe le voile à l'islam, est confondue et déconstruite. Du reste, un tableau de Georges de La Tour est simultanément reconfiguré en fond de scène : la pose, les vêtements de la comédienne et le décor dans lequel elle est assise, immobile derrière un mur de verre, évoque en effet la Madeleine pénitente - du moins, si l'on réexamine a posteriori le tableau, un seul détail différencie l'icône de la peinture d'origine celle qui est représentée sur scène : ici, Madeleine n'est pas voilée, et ses longs cheveux blonds sont librement lâchés sur ses épaules. Par cette élision, la représentation scénique efface la preuve que le voile a été porté par le passé en Occident chrétien : la doxa est ainsi corroborée - pour être mieux déconstruite lorsque, dans un second temps, est enfin révélée la source du texte récité (Figure 1).

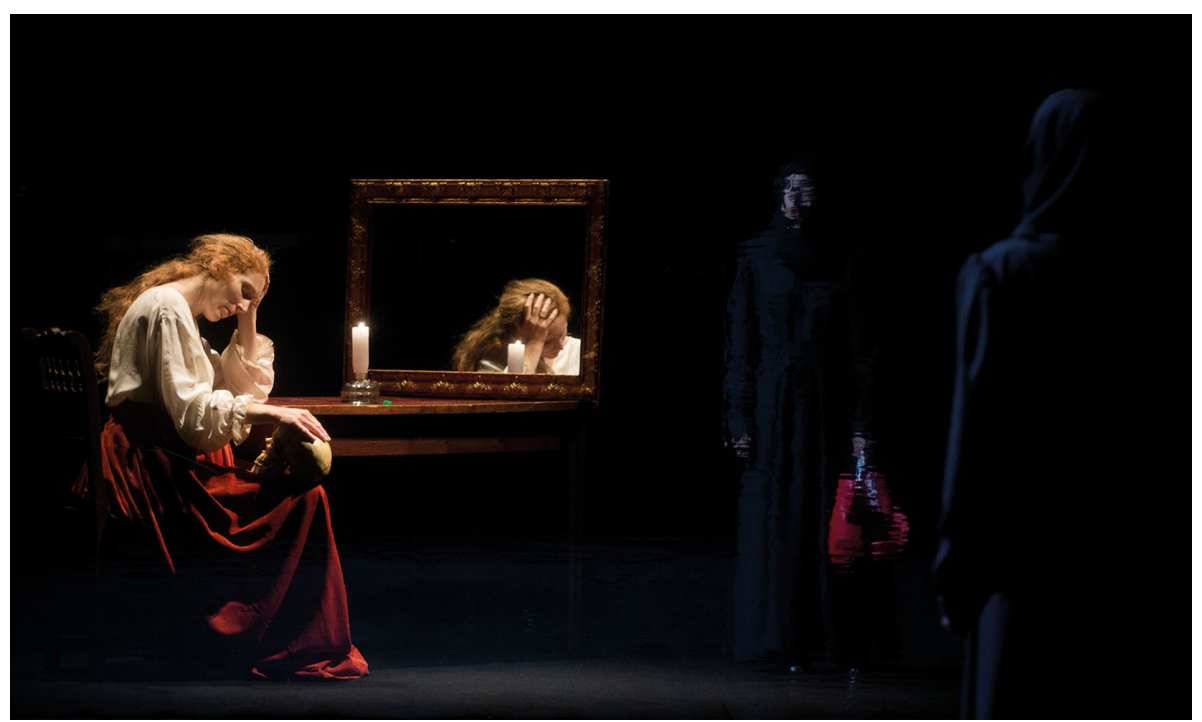

Figure 1 : Reconfiguration de La Madeleine pénitente de Georges de la Tour. (C) Vincent_Arbelet

5 " Toute femme qui prie ou prophétise tête nue fait affront à son chef ; car c'est exactement comme si elle était rasée. Si la femme ne porte pas de voile, qu'elle se fasse tondre. Mais si c'est une honte pour une femme d'être tondue ou rasée, qu'elle porte un voile. L'homme, lui, ne doit pas se voiler la tête : il est l'image et la gloire de Dieu ; mais la femme est la gloire de l'homme. Car ce n'est pas l'homme qui a été tiré de la femme, mais la femme de l'homme, Et l'homme n'a pas été créé pour la femme, mais la femme pour l'homme ". Voir : Traduction Ecuménique de la Bible, I, Corinthiens, XI, 5-8. 
Par la suite, des extraits choisis du film La Rabbia confrontent sur scène des photos de Marylin Monroe et la poésie de Pier Paolo Pasolini, qui livre une vaste critique du monde moderne. Cette séquence dénonce notamment la réification du corps féminin par la société capitaliste en une pure utilité marchande : alors que le voile est, pour le regard occidental, si souvent le signe de l'aliénation de la femme, cet argumentaire est mis en question par la critique de la notion d' "émancipation " féminine, et la dénonciation de l'aliénation que commande l'Occident lui-même - par l'injonction publiciste et pornographique à la nudité et à l'exhibition (Figure 2).

Le monde des magazines, du lancement à échelle mondiale des produits, même humains, est un monde qui tue. Pauvre, tendre Marylin, petite sœur obéissante, accablée par ta beauté comme par une fatalité qui réjouit et tue. Peut-être as-tu pris le bon chemin, nous l'as-tu enseigné. Ton blanc, ton or, ton sourire impudique par politesse, passif par timidité, par respect envers les adultes qui te voulaient ainsi, toi, restée gamine, voilà ce qui nous invite à apaiser la rage dans les pleurs, à tourner le dos à cette réalité maudite, à la fatalité du mal. ${ }^{6}$

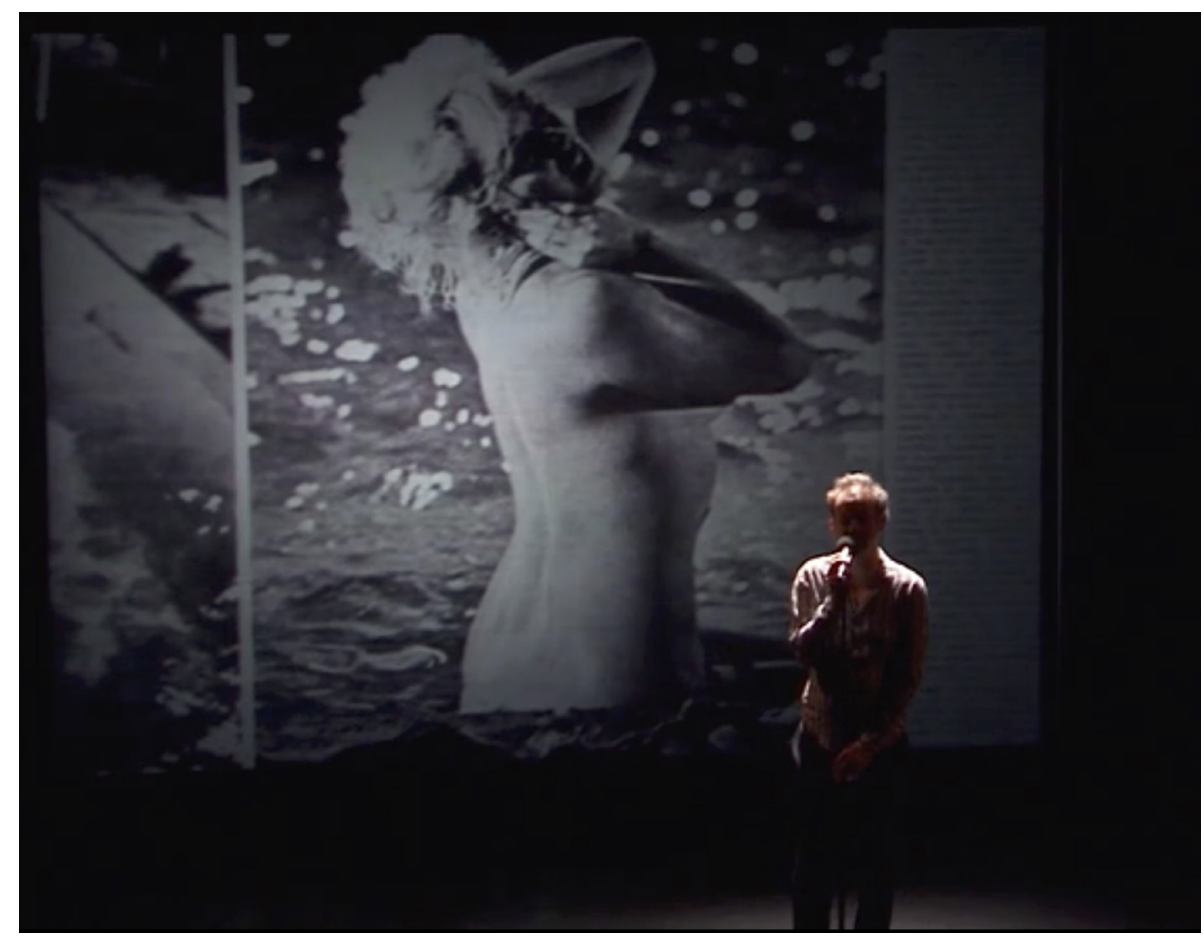

Figure 2 : Photographies de Marylin Monroe, issues de La Rabbia de Pier Paulo Pasolini.

(C) Vincent_Arbelet

6 Ce texte est composé de différents extraits du discours sur la " Normalité " dans La Rabbia (La rage), 1963. 
Par ailleurs, parce qu'il permet de se soustraire au regard et de cesser de faire image, le voile n'apparait pas nécessairement symptomatique d'une oppression, et peut être ainsi considéré au contraire ou comme une marque de pudeur, ou comme la libre protestation contre la réification du corps dénudé.

À côté des images d'archive historiques, sont en outre déployés des documents où le " je " de la parole intime surgit. Les portraits photographiques des grands-mères de la metteure en scène $(1940,1950)$, respectivement ukrainienne et tunisienne et toutes deux voilées, sont notamment présentées : alors qu'il est conventionnellement associé à l'islam, le "voile " n'apparait plus à cet endroit comme un symbole religieux ou idéologique, mais comme un simple fichu ayant régi, par coutume ou utilité pratique, la tenue de femmes d'origines variées. Les photographies, issues des albums de famille, font ainsi éprouver au spectateur une mémoire sensible et une perception intime du voile, qui dénouent l'amalgame dont son port fait souvent l'objet ; là où la presse propage et entérine une interprétation univoque et généralisante du voile, le spectacle rend chaque femme voilée à sa singularité. De la même manière, différentes saynètes donnent corps et voix à des personnages de la fiction romanesque, qui viennent subjectiver et personnaliser les problématiques liées au port du voile. Notons par exemple le transposition dramatique d'un extrait de Vernon Subutex ${ }^{7}$ de Virginie Despentes, dans lequel un homme français, originaire d'Afrique du Nord et indifférent à sa culture d'origine, entre en conflit avec sa fille lorsque celle-ci décide de revêtir le voile par conviction religieuse et politique. À la toute fin du spectacle, différents portraits de femmes voilées sont également présentés par vidéo-projection : ces dernières prennent la parole pour dire leurs expériences personnelles, et témoigner de la diversité des possibilités d'existence du voile - religieuse, politique, ou traditionnelle. Un jeu de regard est alors engagé : les femmes observent tout autant qu'elles sont observées, et paraissent jauger le regard qui est porté sur elle, attentives à la réception de la singularité de leurs expériences - singularité relayée par le plan serré de la vidéo, qui révèle les plus menus détails de leurs visages et de leurs expressions.

Enfin, notons la présence et le traitement de la musique : introduite non comme un chant illustratif mais comme un langage différent, la musique semble en effet posséder une signifiance propre qui vient déplacer le rythme et l'intensité de la parole à laquelle elle est tissée, pour déconstruire les certitudes de son propos. La récitation de L'Épître aux Corinthiens de Saint-Paul en est la parfaite illustration : parce que la parole épouse la structure rythmique de la musique qui l'accompagne, le temps de la diction se dilate, les accents qui structurent la prise conventionnelle de parole sont déplacés, et le propos, étrangéisé, perd sa familiarité et son évidence. À mesure que le tempo accélère et que le son gagne en volume, le ralentissement de la diction et la répéti-

7 Virginie Despentes, Vernon Subutex, Paris, Grasset, 2015. 
tion frénétique du texte aboutissent à un " effet de transe auto-induite ${ }^{8}$ qui rend compte de la fanatisation de la parole dogmatique. En outre, l'humeur musicale est élaborée par le croisement des mélodies à la fois électroniques et traditionnelles, occidentales et orientales : si elle ne porte évidemment pas à elle seule un propos intelligible - et si elle contribue même à désintellectualiser la scène -, la musique instaure toutefois des "conditions atmosphériques " créatrices d'un état affectif propice à la réception de la polysémie des représentations du voile convoquées sur le plateau.

\section{"What you see is what you see »}

En définitive, le spectacle dans son ensemble entraîne le regard à ne plus considérer le voile comme un emblème renvoyant à des réalités extérieures (religieuses et politiques) : ramené à sa simple concrétude objectivable (un tissu posé sur les cheveux) ou à une convention potentiellement universelle, l'objet est dépouillé de toute symbolique revendiquée et perd son caractère distinctif - pour ne se justifier que de son usage pratique (se protéger du soleil) ou traditionnel. "What you see is what you see" : à l'ouverture du spectacle, la comédienne, voilée et face au public, récite et répète à plusieurs reprises l'aphorisme de Frank Stella ${ }^{10}$ (Figure 3).

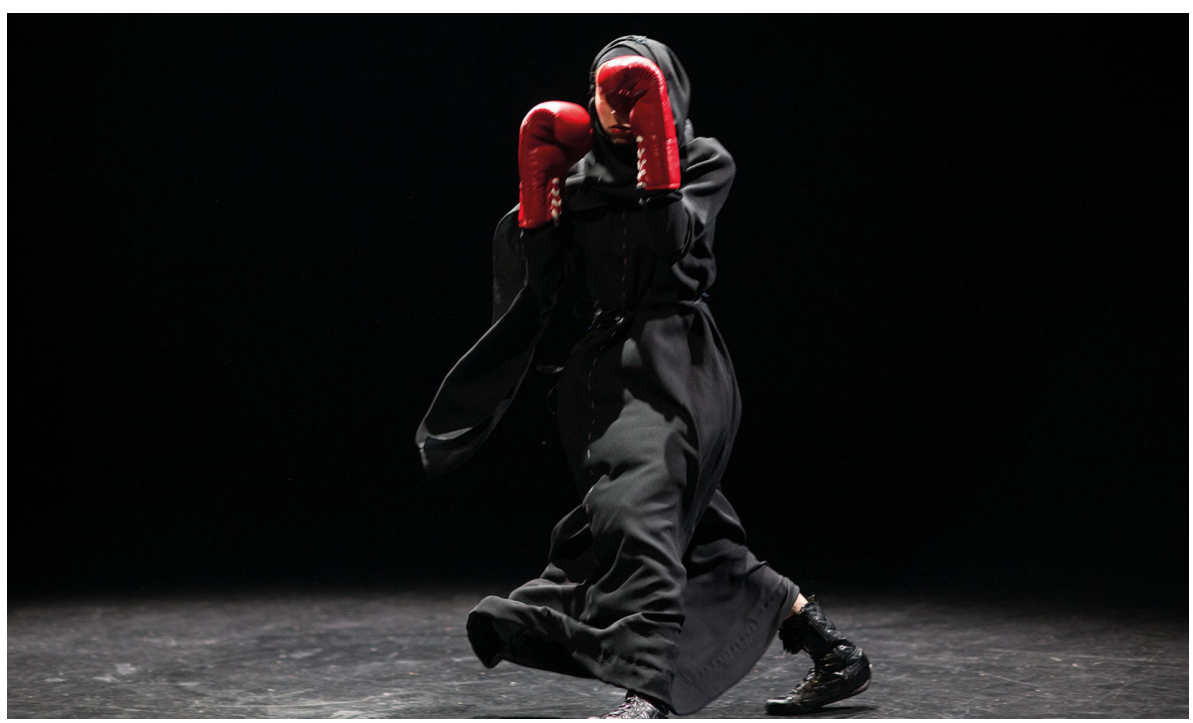

Figure 3 : Voilée intégralement, la comédienne mime un combat de boxe, et ne cesse de répéter l'aphorisme de Franck Stella : "What you see is what you see ». (C) Vincent_Arbelet

8 Entretien téléphonique particulier avec Myriam Marzouki, le 10 novembre 2017.

9 Idem.

10 B. Glaser, "Questions to Stella and Judd ", Art News, 1966, p. 58-59. 
Est alors d'emblée énoncée la réception à la fois attendue et élaborée par le spectacle - un regard "tautologique " ou " analphabète ", et non un regard de "croyance ", dissociant l'objet de son " aura " ${ }^{11}$ que constituent les images et les significations qui s'y rapportent spontanément. Alors que les discours politiques et médiatiques ne cessent de charger symboliquement l'objet, le spectacle propose ainsi d'entraîner le regard à " ne voir que ce qu'il y a à voir " : ni outil de dissimulation, ni nécessaire volonté de parâttre, le voile n'est pas clairement défini, et le signifiant demeure suggestif et ambigu ${ }^{12}$. C'est en ce sens que Georges Didi-Huberman fait la distinction entre des images qui "prennent parti »-qui construisent et transmettent une idéologie pour dire ce qui serait le vrai ou la réalité - et les images qui " prennent position » : ces dernières documentent poétiquement le réel, le document n'étant pas une preuve ou le porteparole d'un parti-pris idéologique, mais une simple mise en scène qui, exposée et déconstruite, déplace les certitudes.

Le montage rend équivoque, improbable, voire impossible, toute autorité de message ou de programme. C'est que dans un montage de ce type, les éléments - images et textes - prennent position au lieu de se constituer en discours et de prendre parti. ${ }^{13}$

L'indécidabilité ${ }^{14}$ alors engendrée n'est donc pas le fait d'une analyse vaine ou aporique qui ne parviendrait ni à formuler ni à définir la réalité : elle est la conséquence d'un regard qui ne cherche pas ce qui serait la juste interprétation du voile, mais questionne au contraire l'univocité des signes et rend l'objet à son ambiguïté. Or, dès lors qu'il ne s'agit pas de cerner ce qui serait la vérité ou le bon usage de l'objet, et dès lors que la symbolique de l'objet est remise en perspective, un rapport d'analogie ou de ressemblance se tisse entre les différentes possibilités d'existence du voile; bien qu'ils soient relatifs à l'inscription sociale et culturelle du sujet, ces usages au départ hétérogènes sont réconciliés par le montage documentaire en une « relation plus fondamentale de co-appartenance ", au sein d'un "même tissu référentiel ${ }^{15}$ ". En déterrito-

11 Le « regard de croyance » est proche de celui d'« aura » que Walter Benjamin définit dans L'homme, le langage et la culture. Essais (Paris, Denoël Gonthier, 1974) : selon lui, la seule perception d'un objet, structurée par un imaginaire construit culturellement, charrie un ensemble de significations qui déterminent son interprétation immédiate. Nous renvoyons à Georges Didi-Huberman, Ce que nous voyons, ce qui nous regarde, Paris, Minuit, 1992. Notons que Georges Didi-Huberman a largement contribué à la dramaturgie du spectacle dans son ensemble.

12 Assia Djebar, Ces voix qui miassiègent... en marge de ma francophonie, Paris, Albin Michel, 1999, p. 43

13 Georges Didi-Huberman, Quand les images prennent position, Paris, Minuit, 2009, p. 118.

14 Notons peut-être une exception, qui est la critique de la réification moderne et occidentale du corps féminin. Le spectacle adopte en effet une idéologie claire, qui va à l'encontre d'une autre rhétorique féministe : le voile n'est pas davantage une oppression patriarcale systémique que ne l'est l'injonction au dévoilement - dévoilement qui n'est nullement le signe d'une libération sexuelle ou de la libre volonté de la femme.

15 Jacques Rancière, Le Destin des images, Paris, la Fabrique, 2003, p. 66. 
rialisant nos perceptions et en ouvrant les possibilités de regard, le spectacle prend donc le contre-pied du débat politique et médiatique qui révèle un désir hystérique et toujours renouvelé de définir l'objet : à l'inverse, il apaise la crispation sociale que l'objet polarise, nuance le clivage qui divise la communauté, et crée les conditions d'une véritable écoute entre les forces en conflit.

\section{Le regard: la fabrique de l'altérité}

Le spectacle ne nie pas la charge symbolique dont le voile peut être volontairement l'écrin, mais démontre plutôt que son interprétation est indécidable et trop hâtivement déterminée : le port du voile est-il un libre choix, ou le signe d'une oppression patriarcale systémique ? Est-il commandé par une conviction religieuse, par la revendication d'une identité adversaire, ou par le simple respect d'une habitude vestimentaire devenue tradition, respectée par coutume, et politiquement neutre?

La question n'est pas là, et n'est pas finalement celle que pose le spectacle. Il ne s'agit pas de statuer sur ce qui motive le port du voile, mais de révéler la manière dont notre propre regard impose spontanément au voile une symbolique identitaire et politique. Les unes médiatiques qui jalonnent le spectacle révèlent notamment à quel point les représentations qui nourrissent notre imaginaire renvoient sans cesse le voile à la burqa (dont le port reste très minoritaire en France), et fait ainsi de l'objet le signe d'une différence culturelle, religieuse ou politique, adversaire et à combattre. Or, c'est précisément par cette altérisation que l'objet est devenu a posteriori un réel enjeu identitaire : le spectacle donne notamment l'occasion de se souvenir que l'interdiction du voile à l'école et dans l'espace public n'a pas aidé à favoriser la mixité sociale, mais a conduit à la déscolarisation des jeunes filles voilées et au développement des écoles confessionnelles - et, donc, au repli identitaire pourtant initialement combattu. Des photographies et affiches rappellent également la violente campagne coloniale de dévoilement menée en Algérie française au début des années 1950 : l'entreprise avait paradoxalement conduit les femmes dévoilées à prendre le voile, l'objet étant devenu un symbole de lutte politique contre l'oppression coloniale, et signe de solidarité entre concitoyennes. Comme souvent lors des processus de marginalisation, la stigmatisation du voile sert donc à la fois des velléités oppressives et des revendications émancipatrices : pour les dominants, elle structure l'exclusion d'une catégorie de la population ; pour les sujets opprimés, l'appropriation de la stigmatisation, et la revendication des significations identitaires et politiques du voile (potentiellement fabriquées par la puissance dominante) apparaissent comme une stratégie pour lutter contre l'acculturation (Figure 4). 


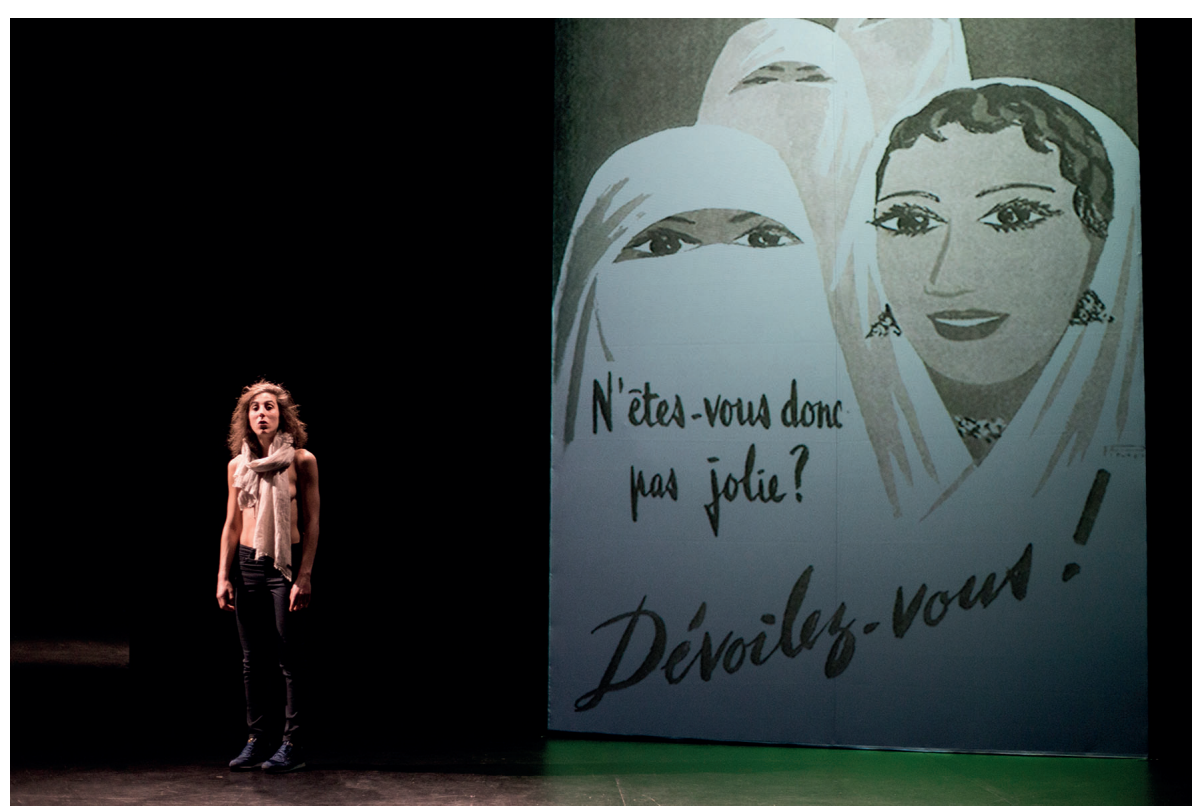

Figure 4 : Affiche de promotion de la violente campagne de dévoilement en Algérie française.

(C) Vincent_Arbelet

Plus proche du " montage symbolique » que du " montage dialectique " ${ }^{16}$, le spectacle maintient donc une forme de méfiance envers la réalité à laquelle le document est censé fournir un accès immédiat : plutôt que de prendre acte du potentiel écart entre nos imaginaires et le réel objectif dont le document serait la preuve, le spectacle analyse, repense et considère le document comme une représentation subjective qui construit le monde davantage qu'il n'informe. Les fonctions référentielles du document sont alors interrogées, et le style interne qui l'élabore (procédés narratifs, rhétoriques et stylistiques) est révélé, mettant en faillite la construction des effets de sens (fables, mythes et métaphores) qui structurent notre saisie du monde ${ }^{17}$; à la fois écrin et écran, interprétation et suspension de l'analyse et de la critique, ces effets de sens, intériorisés, donnent accès à la réalité tout autant qu'ils en " obturent l'accès " ${ }^{18}$.

Plutôt que de procéder à la présentation chronologique ou logique du fait politique, le spectacle ne procède pas à une démonstration ou à une mise en forme objective de la réalité, mais préfère simplement dépayser notre rapport aux documents, pour révéler et subvertir les opérations discursives et les habitudes

16 Jacques Rancière, Le Destin des images, op. cit., p. 66.

17 À ce sujet, voir : Armelle Talbot, "Poétique du document et poétisation documentaire : L'Instruction de Peter Weiss ", in Marianne Bouchardon et Florence Naugrette (éds), La Poésie dans les écritures dramatiques contemporaines, Paris, Classiques Garnier, p. 118. 
perceptives qui fabriquent le réel - confondant ainsi les potentiels préjugés, stéréotypes ou raccourcis qui peuvent structurer notre appréhension du voile.

L'on rétorquerait, à celles et ceux qui soutiennent que l'apaisement social à laquelle œuvre le spectacle implique l'élaboration d'un consensus improductif, que l'élargissement du regard que le spectacle propose altère au contraire la doxa imposant à l'objet une symbolique univoque - et, au sujet, la seule attitude binaire qu'est ou l'apologie ou le réquisitoire. Alors que l'interprétation du voile est trop souvent tributaire d'un amalgame construit socialement, le spectacle propose donc un regard propice à l'érosion puis à la métamorphose de la réception du voile, alors plurielle et conséquemment dissensuelle.

Chloé Dubost

Doctorante contractuelle

Université Toulouse-Jean Jaurès chlo3-d@hotmail.com

\title{
Résumé
}

L'enjeu de Ce qui nous regarde, spectacle documentaire de Myriam Marzouki, est de révéler la manière dont le regard porté sur le voile est fabriqué et déterminé socialement, par l'intériorisation de différentes narrations médiatiques et politiques qui associent d'emblée l'objet à une symbolique religieuse, politique ou identitaire. La poésie documentaire, née de l'articulation et de la friction entre différentes textures documentaires, déconstruit donc les effets de sens des représentations conventionnelles, proposent des images inédites qui dénouent l'amalgame, et contribue ainsi à l'avènement d'un nouveau rapport affectif et symbolique à l'objet - alors rendu à sa polysémie et à son indécidabilité.

\section{Mots-clés}

Théâtre documentaire, Montage symbolique, Postcolonialité, Fabrique de l'altérité, Emancipation, Féminisme, Croyance.

\begin{abstract}
The purpose of Ce qui nous regarde, a Myriam Marzouki's documentary play, is to reveal how the occidental point of view on the hijab is produced and socially determined. It shows how the political discourse, reused and strengthened in the media, tends to associate systematically the veil with religious, political and identity significations. The documentary poetry, stemmed from various documentary textures, manages to subvert the conventional significations and suggests new images, which unravels the confusion and the amagalms. In this way, the play contributes to the renewal the emotional and symbolic relationship with the veil-legitimately polysemic and indeterminate.
\end{abstract}

\section{Keywords}

Symbolic editing, Postcolonial, Alterity make, Emanciaption, Feminism, Belief. 